\title{
IMAGE SEGMENTATION BASED ON GRAPH-CUT MODELS AND PROBABILISTIC GRAPHICAL MODELS: A COMPARATIVE STUDY
}

\author{
MAEDEH BEHESHTI, ALAN WEE-CHUNG LIEW \\ School of Information and Communication Technology, Gold Coast Campus, Griffith University, QLD 4222, Australia \\ E-MAIL: maedeh.beheshti@griffithuni.edu.au, a.liew@griffith.edu.au
}

\begin{abstract}
:
Image Segmentation has been one of the most important unsolved problems in computer vision for many years. Recently, there have been great efforts in producing better segmentation algorithms. The purpose of this paper is to introduce two recently proposed graph based segmentation methods, namely, graph-cut models (deterministic) and unified graphical model (probabilistic). We present some foreground/background segmentation results to illustrate their performance on images with complex background scene.
\end{abstract}

\section{Keywords:} Models

Image Segmentation; Graph-cut segmentation; Graphical

\section{Introduction}

Partitioning an image into meaningful parts or regions that share certain visual characteristics based on image attributes like grey level, colour, texture, depth or motion is the main purpose of image segmentation. Image segmentation plays a crucial role in many applications such as object identification and recognition, content based image retrieval, and medical image analysis. In spite of the many existing algorithms and techniques for image segmentation, in this paper we focus only on graph partitioning methods. Graph based segmentation, which is based on different principles to many traditional segmentation algorithms, is a relatively new segmentation method. Graph based segmentation algorithms can be broadly divided into two types 1- deterministic 2- probabilistic [1]. Algorithms such as $\mathrm{max} / \mathrm{min}$ cut and normalized cut belong to the deterministic approach, and algorithms such as Markov Random Field and Bayesian Network belong to the probabilistic graphical approach. Energy minimization and probabilistic inference are the main tasks in graph cut algorithms and probabilistic graphical models, respectively. Because of dealing with many variables, the energy function of graph-cut algorithms tends to be very complex and often NP-hard, especially for non-convex problem [2][3][4].
In this paper we describe two representative graph-based segmentation algorithms, one from each type. We describe the idea behind each method, and present some experimental evaluation to show their empirical performance.

\section{GRAPH-CUT SEGMENTATION ALGORITHMS}

\subsection{Binary and Multi-Label Graph-cut}

Boykov and Jolly [2] proposed a graph-cut method for segmentation which involves finding the s-t cut of minimal total cost with 2 labels (binary labels) in a graph. In their approach, an image is to be segmented into object and background, with soft constraints on boundary and region. In their model, an undirected graph $G(V, E)$ (see Figure 1) is defined in which $V$ and $E$ represent graph nodes (equal to image pixels $P$ ) and graph edges, respectively. In addition, two extra terminal nodes, $S$ (source) and $T$ (sink), which represent the object and background labels, are also defined. All edges in this graph are divided into two classes: n-links and t-links, where n-links are edges between pixels and $\mathrm{t}$-links are edges between pixels and terminal nodes.

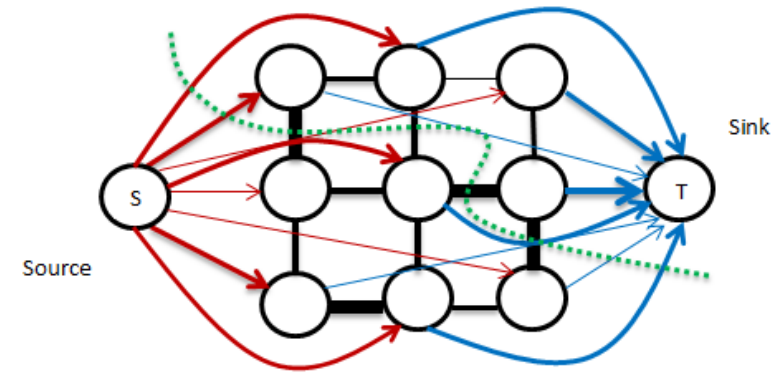

Figure 1. A graph with two terminals - Arrows show the flow from source (S) to sink $(\mathrm{T})$ - dashed curve represents the min-cut point [2][3]. - Thickness of the links represent the amount of flows that has been sent from the source to the destination

Each non-terminal node has two terminal links, one for $S$ and one for $T$, and a number of n-links. The n-links or neighborhood links are the links between pixels and they 
construct an arbitrary neighborhood system. Each edge has associated with it a cost or weight. An optimal s-t cut is a subset of edges with minimum cost which partition the graph (image) into two parts, source and terminal. In this regard, the purpose of segmentation is to minimize the energy function in (1) which is the sum of regional (cost of t-links) and boundary terms (cost of n-links).

$$
E(A)=\delta \cdot \operatorname{Region}(A)+\operatorname{Boundary}(A)
$$

In (1), $A$ defines a segmentation and $\delta \geq 0$ is a coefficient which emphasizes the region term. The segmentation is done based on max flow algorithm [5] which we will elaborate in section 2.4.

To handle more than two labels, i.e. $|L| \geq 3$, multi-label energy minimization through multi-terminal min-cut or move-making (local search) algorithms like alpha-beta swap algorithm and alpha expansion was proposed [4]. Alpha-beta swap algorithm and alpha expansion are two kinds of very large search neighborhood techniques (VLSN). Through VLSN algorithms and appropriate move space, an exploration of an exponential number of alternative labeling will be facilitated in polynomial time. Due to the lack of space we only consider $\alpha \beta$-swap here. Given current labeling $f$ from a pair of labels $\{\alpha, \beta\} \in L$ the purpose is to find a labeling $f^{\wedge} \in\{\alpha, \beta\}$ that minimizes the energy function in equation (2) [4]. According to Figure 2 each variable labeled either $\alpha$ or $\beta$ is able to keep its current label (See Figure 2.a) or swap to the other labels. Figure 2.b represents the swap between $\alpha, \beta$ labels, and variables with label $\beta$ have been expanded and variables with label $\alpha$ have been decreased. In addition, Figure 2.c represents the swap between $\beta, \gamma$ labels where variables with label $\beta$ have been decreased and variables with label $\gamma$ have been increased. Each time only two variables from move space will be selected for swapping. If there are $K 1$ variables with a current label in $\{\alpha, \beta\}$ there will be $2^{K 1}$ possible $\alpha \beta$-swap moves available. Consider the following energy function

$$
\begin{gathered}
\operatorname{Energy}(f)= \\
\operatorname{Energy}_{\text {smooth }}(f)\left[\sum_{\{p, q\} \in N} V_{p, q}\left(f_{p}, f_{q}\right)\right]+ \\
\operatorname{Energ}_{\text {data }}(f)\left[\sum_{p \in P} D_{p}\left(f_{p}\right)\right]
\end{gathered}
$$

where $N$ is the set of interacting pairs of pixels such as $p$ and $q, D_{p}\left(f_{p}\right)$ is the cost of assigning a label such as $l$ to a pixel $p$, and $V_{p, q}\left(f_{p}, f_{q}\right)$ is a smooth term between two pixels $p$ and $q$ that shows coherence and consistency between labels [3][4]. The first part in equation (2) measures the extent to which $f$ is not piecewise smooth while the second part measures the disagreement between $f$ and the observed data [4]. The smooth term can be viewed as a regularization term as it reduces inconsistency and fluctuations caused by noisy data.

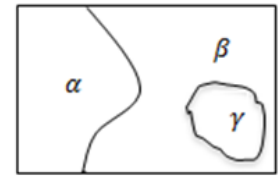

(a)

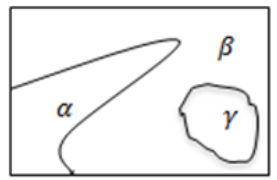

(b)

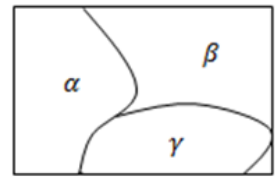

(c)
Figure 2. The left figure shows current labeling containing three $\alpha, \beta$ and $\gamma$ parts - The middle and right figures show an $\alpha, \beta$ and $\gamma, \beta$ swap respectively.

\subsection{Optimal Multi-Region Object Segmentation}

In the optimal multi-region model of [6], one layer is used per region and interaction between regions is encoded into the energy function. Besides the usual data term and smooth term in equation (2), there is an inter-region interaction term in the energy function in (3).

$$
\begin{gathered}
\operatorname{Energy}(f)= \\
\text { Energy }_{\text {smooth }}(f)\left[\sum_{\{p, q\} \in N} V_{p, q}\left(f_{p}, f_{q}\right)\right]+ \\
\text { Energy }_{\text {data }}(f)\left[\sum_{p \in P} D_{p}\left(f_{p}\right)\right]+\sum_{\substack{m, n \in L \\
m \neq n}} W^{m n}\left(x^{m}, x^{n}\right) \\
W^{m n}\left(x^{m}, x^{n}\right)=\sum_{p q \in N^{m n}} W_{p q}^{m n}\left(x_{p}^{m}, x_{q}^{n}\right) \quad \text { is a }
\end{gathered}
$$
geometric term for making interaction between two regions $m$ and $n$ (two superscripts in $W$ ), and $x_{p}^{k}=1$ means pixel $p$ is included in region $k, x_{p}^{k}=0$ means pixel $\mathrm{p}$ belongs to the background. There are three types of geometric constraints that are applied to the constructed graph: containment, exclusion and attraction. Containment constraint requires that region $\mathrm{B}$ has to be included in region A. Exclusion constraint requires that $\mathrm{A}$ and $\mathrm{B}$ cannot overlap at any pixel. Attraction requires that region A should not be grown too far away from region B. Figure 3 shows how the constraint energy terms between two regions.

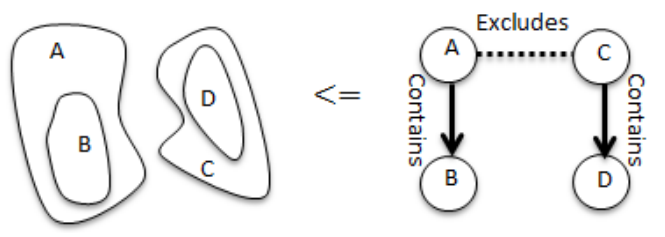

Figure 3. An example of two mutually exclusive regions [6]

This multi-region model has been successfully applied to medical image segmentation. All graph-cut based segmentation algorithms suffer from some difficulties in the optimization procedure. These difficulties in some cases stem from the maximum flow/minimum cut algorithms which act 
as the main optimization techniques behind the graph-cut algorithms.

\subsection{Maximum-Flow/Minimum-Cut Algorithms}

Maximum flow algorithms are the basis of many global optimization methods. There are many methods for implementing the maximum flow algorithms, such as Goldberg-Tarjan's [5] "push-relabel" methods and algorithms based on Ford-Fulkerson [7]. In maximum flow algorithms, given a weighted graph where the weight of an edge represents the capacity of the edge, we want to determine the maximum amount of flow that we can send from one vertex $S$ (source) to another vertex $T$ (sink) in the graph, according to the illustration in Figure 1. Locality and parallelization are two important factors in flow propagation in $\mathrm{max} / \mathrm{min}$ algorithms. Locality refers to fitting the massive and huge graph vertices into computer memory, and parallelization refers to carrying out the steps in maximum flow algorithm asynchronously [5][8]. Absence of each of them will result in an impractical maximum flow algorithm. In addition, the running time of any maximum flow is an important issue that has to be considered. On one hand, finding the shortest path for sending flow is the main goal. On the other hand, looking for the shortest path is expensive and time consuming.

\section{UNIFIED GRAPHICAL MODELS AND IMAGE SEGMENTATION}

In graph-cut approaches prior knowledge about image data are usually not considered. In order to overcome this weakness, Zhang et al. [1][9] proposed a combinatory probabilistic framework of a directed (e.g. Bayesian Network) and an undirected (e.g. Conditional Random Field (CRF) or Markov Random Model (MRF)) graphical models. It is inspired by human's ability to segment an image with additional constraints such as contour smoothness, connectivity, object shape, etc. In contrast to graph-cut models which utilize an energy function, probabilistic graphical models perform probabilistic inference for segmentation.

Probabilistic graphical models provide easy visualization of the structure of a probabilistic model [1][9]. In a probabilistic Graphical Model, each node represents a random variable or group of random variables and the links show probabilistic relationships between these variables. In this regard, the whole graph expresses the way in which the joint distribution over all of the random variables can be decomposed into a product of factors.

Many methods have been proposed for image segmentation based on MRF, CRF, Bayesian Network (BN) and their combination, such as the MRF hybrid model of
Hinton et al. [10], the BN and MRF model of Murino et al. [11] and Liu et al. [12]. In the recent method by Zhang and Ji [1], the causal and non-causal relationships among all the image entities are considered. According to Figure 4, it tries to facilitate region-based (upper cloud) and edge-based (lower) segmentation in an image by unifying CRF and BN. This approach consists of three parts, CRF, BN and a combination of these two models. The CRF model (undirected) is constructed based on a superpixel model. Superpixels are specified by an over-segmentation preprocessing task on the image that extracts some homogeneous parts (regions). Each part is equal to one region node in CRF.

The model is a multi-layer model which supposed the $\mathrm{CRF}$ as a labeling system that assigns a label based on local feature vector extracted from image $(\mathrm{x})$ for a superpixel $(\mathrm{Y})$ and $\mathrm{BN}$ as an inference system of the type of the edge nodes given various measurements and constraints. CRF part plays the region-based image segmentation role and $\mathrm{BN}$ part tackles edge-base segmentation. Because of the inability of CRF in representing causal model, Bayesian Network is deployed to model the causal relationship. BN part provides a representation of the relationships between superpixels (Y), the edge segments (e) and vertices (v). The model has been represented for 4 superpixels in Figure 4. Vertices (v) are a place where three or more edges intersect and $\mathrm{M}$ denotes measurements of features of edges (e) and vertices (v).

In the next section, we perform some comparative study to illustrate the performance of the two approaches.

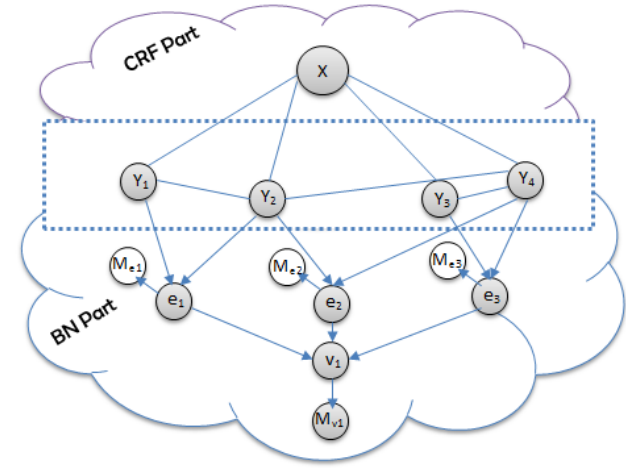

Figure 4. A structure for Unified Graphical Model combining CRF mode and $\mathrm{BN}$ model by region nodes [1]

\section{EXPERIMENTS}

We perform segmentation on the Weizmann (horse) dataset [13][14]. Weizmann horse dataset includes color images of 328 different side-views of horses in addition to their ground truth masks. Because of the variety of images with different appearances and side views, this is a 
challenging dataset for segmentation. We compare the unified graphical model work and multi-label graph-cut [13] on a group of five, $320 * 213$ pixels images of this dataset. Figure 5 shows examples of horse images in color and their binary segmentation results from unified graphical model [1] and graph-cut model [13]. Our results show that for object extraction or foreground/background segmentation, unified graphical model has better performance. The segmentation accuracy is computed using Dice index as given in equation (4). In case of foreground/background we have $n=1$.

$$
\operatorname{Dice}(R, S)=\frac{1}{n} \sum_{1}^{n} \frac{2|R \cap S|}{|R|+|S|}
$$

Here, $\mathrm{R}$ denotes the ground truth, $\mathrm{S}$ denotes the segmented image and $\mathrm{n}$ is the number of segmented areas. The results of graph-cut model show that this model does not have a good performance on images with one object and complicated background. In particular, when there is common color between foreground and background or in the case of the same intensity the model will not show good results.

We also performed another experiment on a group of 5 gray-scale images of Weizmann (horse) dataset to show the robustness of these two methods for precise segmentation without color information. Figure 6 shows examples of horse images in gray-scale and their binary segmentation results from unified graphical model [1] and graph-cut model [13]. In this case also unified graphical model has better performance than graph-cut model. Nevertheless it can be seen in Figure 5 and Figure 6 that there is a huge difference between graph-cut accuracy results for color images and graph-cut accuracy results for gray-scale ones. Because the main basis of graph-cut approach is based on intensity differences, the performance for images with large intensity difference between foreground and background is much better than the others.

In terms of speed, the computation cost is $O\left(m n^{2}|C|\right)$ [7] for graph-cut, where $m$ and $n$ are the number of edges and nodes, respectively, and $|C|$ is the cost of minimum cut for graph-cut model. In unified graphical model the time complexity is dependent on the constructed graph. The graph usually consists of 700 to 1500 nodes [1]. For images of the same size, segmentation time complexity for graph-cut model is usually less than that of unified graphical model.

\section{CONCLUSIONS}

In this paper, we described two graph-based approaches to image segmentation, namely, graph-cut approach and probabilistic graphical modeling approach and compared their performance on several images from one popular dataset.
Our results show that for images with complicated background, the unified graphical model which is able to take into account multiple prior constraints have superior performance.

\section{References}

[1] L. Zhang and Q. Ji, "Image segmentation with a unified graphical model," Pattern Analysis and Machine Intelligence, IEEE Transactions on, vol. 32, pp. 1406-1425, 2010.

[2] Y. Boykov and M.-P. Jolly, "Interactive graph cuts for optimal boundary \& region segmentation of objects in ND images," in Computer Vision, 2001. ICCV 2001. Proceedings. Eighth IEEE International Conference on, 2001, pp. 105-112.

[3] Y. Boykov and G. Funka-Lea, "Graph cuts and efficient ND image segmentation," International Journal of Computer Vision, vol. 70, pp. 109-131, 2006.

[4] Y. Boykov, O. Veksler, and R. Zabih, "Fast approximate energy minimization via graph cuts," Pattern Analysis and Machine Intelligence, IEEE Transactions on, vol. 23, pp. 1222-1239, 2001.

[5] A. V. Goldberg and R. E. Tarjan, "A new approach to the maximum-flow problem," Journal of the ACM (JACM), vol. 35, pp. 921-940, 1988.

[6] A. Delong and Y. Boykov, "Globally optimal segmentation of multi-region objects," in Computer Vision, 2009 IEEE 12th International Conference on, 2009, pp. 285-292.

[7] Y. Boykov and V. Kolmogorov, "An experimental comparison of min-cut/max-flow algorithms for energy minimization in vision," Pattern Analysis and Machine Intelligence, IEEE Transactions on, vol. 26, pp. 1124-1137, 2004

[8] A. Delong and Y. Boykov, "A scalable graph-cut algorithm for ND grids," in Computer Vision and Pattern Recognition, 2008. CVPR 2008. IEEE Conference on, 2008, pp. 1-8.

[9] L. Zhang, Z. Zeng, and Q. Ji, "Probabilistic image modeling with an extended chain graph for human activity recognition and image segmentation," Image Processing, IEEE Transactions on, vol. 20, pp. 2401-2413, 2011.

[10] G. E. Hinton, S. Osindero, and K. Bao, "Learning causally linked markov random fields," in AI \& Statistics, 2005.

[11] C. Regazzoni, V. Murino, and G. Vernazza, "Distributed propagation of a-priori constraints in a Bayesian network of Markov random fields," IEE Proceedings I (Communications, Speech and Vision), vol. 140, pp. 46-55, 1993. 
[12] F. Liu, D. Xu, C. Yuan, and W. Kerwin, "Image segmentation based on Bayesian network-Markov random field model and its application to in vivo plaque composition," in Biomedical Imaging: Nano to Macro, 2006. 3rd IEEE International Symposium on, 2006, pp.
141-144.

[13] http://www.wisdom.weizmann.ac.il/ bagon.

[14] E. Borenstein and S. Ullman, "Learning to Segment," Proc. European Conf. Computer Vision, pp. 1-8, 2004.
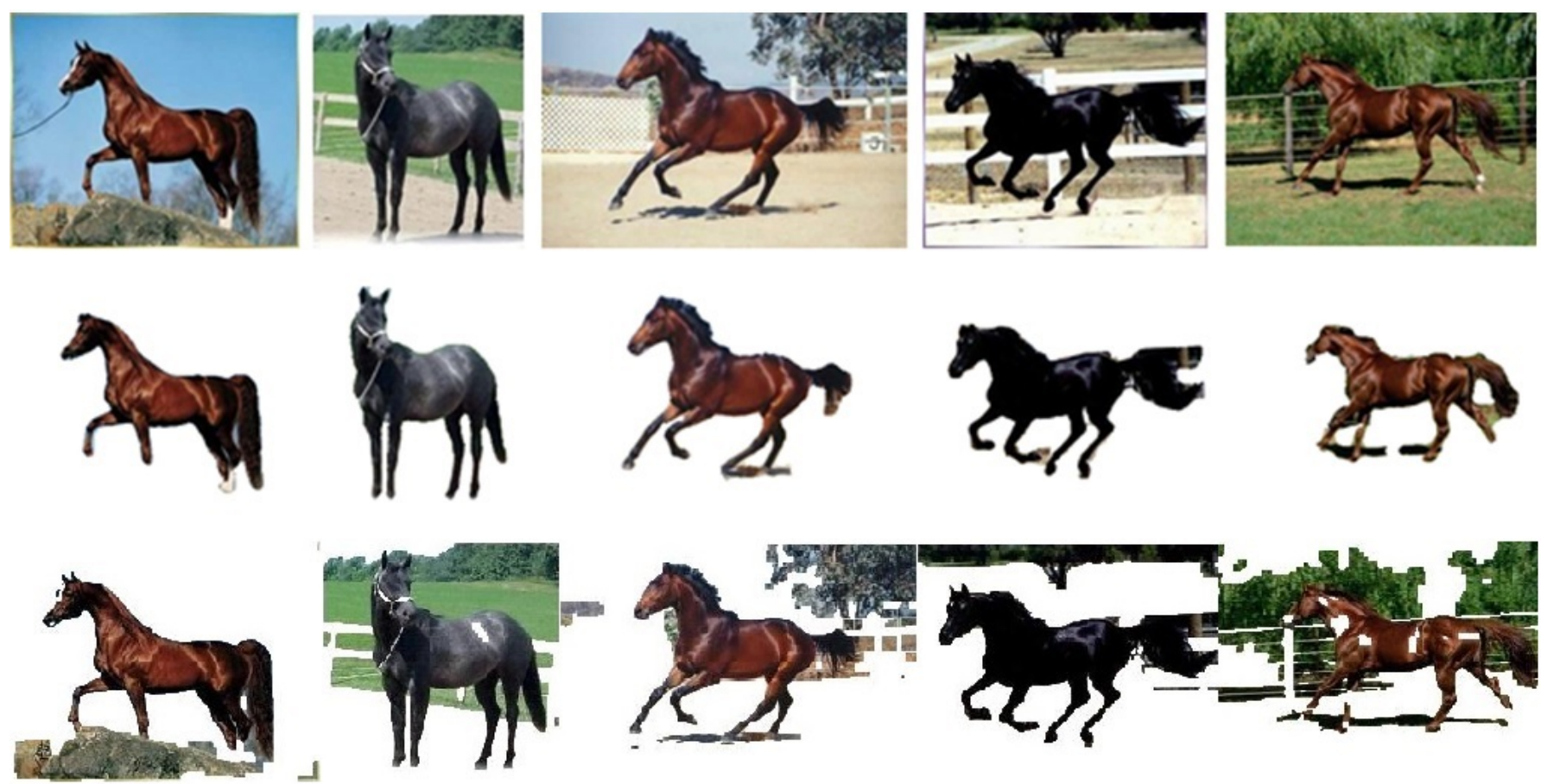

Figure 5. Comparison between unified graphical model and graph-cut model for the horse dataset - The top row shows original images in color, the middle row is the results of unified graphical model [1] with $96.6 \% \mathrm{t}$ accuracy and the bottom row is the results of graph-cut model [13] with $47.8 \%$ accuracy. 

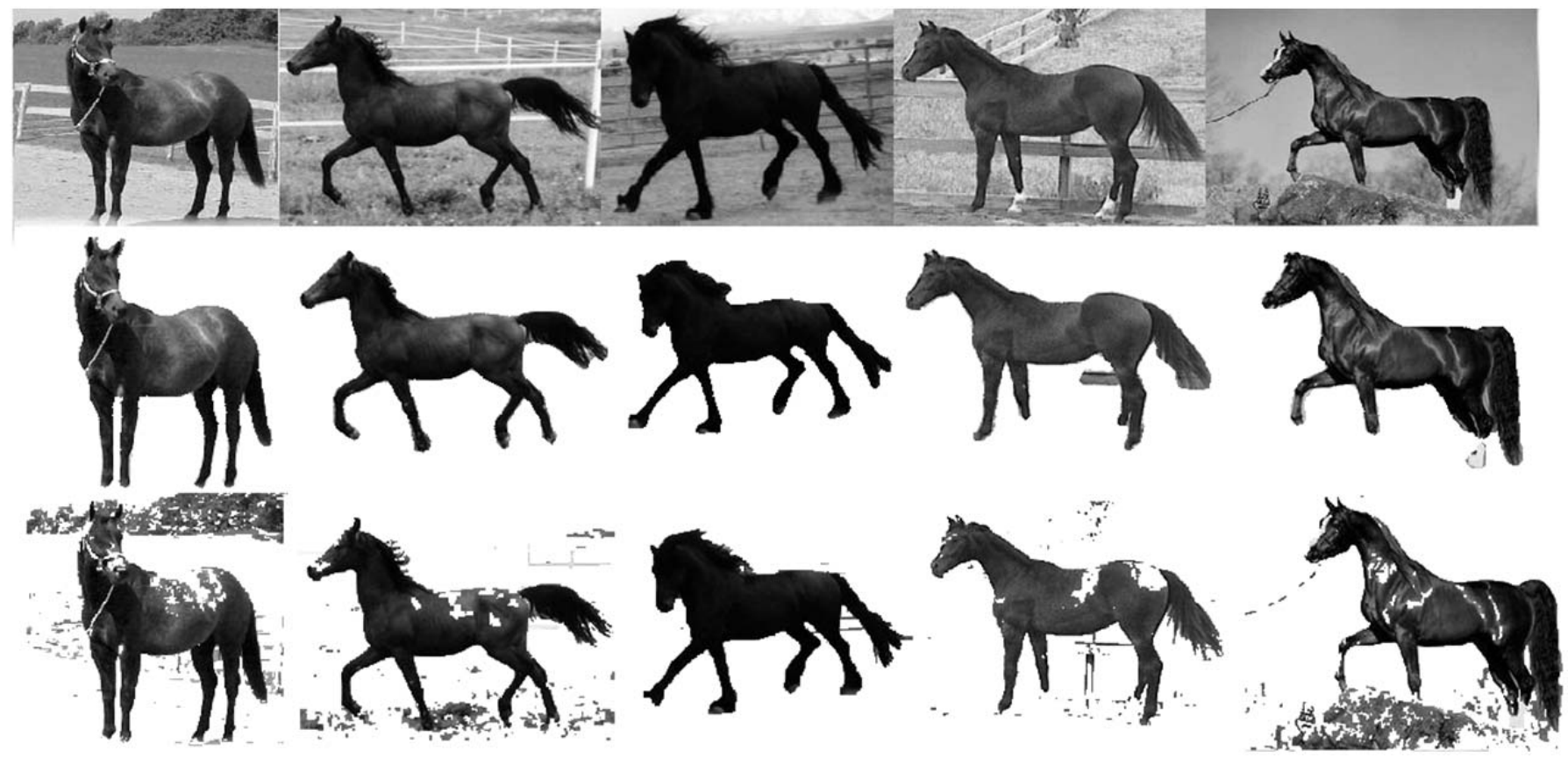

Figure 6. Comparison between unified graphical model and graph-cut model for the gray-scale horse dataset - The top row shows original images in gray-scale, the middle row is the results of unified graphical model [1] with $98 \%$ accuracy and the bottom row is the results of graph-cut model [13] with $91 \%$ accuracy. 